\title{
The Capacity of the Quantum Multiple Access Channel
}

\author{
Andreas Winter
}

\begin{abstract}
We define classical-quantum multiway channels for transmission of classical information, after recent work by Allahverdyan and Saakian. Bounds on the capacity region are derived in a uniform way, which are analogous to the classically known ones, simply replacing Shannon entropy with von Neumann entropy. For the single receiver case (multiple access channel) the exact capacity region is determined. These results are applied to the case of noisy channels, with arbitrary input signal states.

A second issue of this work is the presentation of a calculus of quantum information quantities, based on the algebraic formulation of quantum theory.
\end{abstract}

Keywords - quantum channel, multiway channel, coding, capacity.

\section{INTRODUCTION}

Classical multiway channels were already studied by Shannon [16]. Ahlswede [1], [2] first determined the capacity region of the channel with $s$ senders and $r$ receivers, 'where all senders want to transmit independent messages, which all receivers should get. For a good overview on multiuser communication theory in general consult [6], or the textbook [5].

In the present paper we define the corresponding quantum channel (after Allahverdyan and Saakian [3]), extending the definition of a classical-quantum channel (see [8]). Our motivation is twofold: in the first place, it is a very common situation that many users want to communicate via the same transmission system, and all real systems should be described by quantum mechanics. Then, sec'ondly, we feel that it helps understanding quantum communication if we try to solve questions known in a classical context for quantum channels. This the more, as for Holevo's results on quantum channels (coding theorem and information bound) not only the question and its answer, but even the method of solution is rather close to classically well known mathematics (see [18]), and we should find out whether this similarity extends further.

The results of the present work are: we bound the capacity region, the actual bounds being obtainable from the classical case by formally replacing Shannon entropy by von Neumann entropy in the expressions, thus following a general principle or feeling in physics. The central result is a proof of the direct coding theorem for the multiple access channel (one receiver: $r=1$ ), using the technique of Holevo [9] and Schumacher/Westmoreland [15], which was designed to solve the single-sender case.

The author is with Fakultät für Mathematik, Universität Bielefeld, Postfach 100131, 33501 Bielefeld, Germany. Electronic address: winter@mathematik. uni-bielefeld.de. Research supported by the Deutsche Forschungsgemeinschaft via SFB 343 "Diskrete Strukturen in der Mathematik".
The outline of the paper is as follows: in section [1] the basic definitions are stated, in particular quantum multiway channels are formally introduced. Section III reviews notation and facts about quantum information quantities we shall need. In the following section IV we prove the outer bounds for the capacity region. Sections $\mathrm{V}$ contains a central result on the state disturbance of a measurement with high success probability. In section $\mathrm{VI}$ this result is used to prove the direct coding theorem for the quantum multiple access channel. In the last section VII we comment on the quantum-quantum multiway channel which may be fed with arbitrary input states.

The results of the present work are part of the author's Ph.D. thesis [17], mainly chapter III (with alternative proofs), and appendix A.

\section{Quantum Multiway Channels}

This is the simplest situation of multi-user communication in general: consider $s$ independent senders, sender $i$ using a (finite) alphabet $\mathcal{X}_{i}$, say with an a priori probability distribution $P_{i}$. This alphabet serves as a set of tags of different actions each user may take, such that a signal appears in the output system, composed of the effects of these $s$ independent choices, and the channel noise. To the output system the $r$ receivers have partial access, and their task is to each reconstruct the $s$ messages the senders chose to send. This is to be achieved by block-coding and via a previously agreed coding/decoding scheme.

Formally, this model is captured as follows: the channel is simply a map

$$
W: \mathcal{X}_{1} \times \cdots \times \mathcal{X}_{s} \rightarrow \mathfrak{S}(\mathfrak{Y})
$$

from the input alphabets into the set $\mathfrak{S}(\mathfrak{Y})$ of states of the (finite dimensional) $\mathrm{C}^{*}$-algebra $\mathfrak{Y}$, mapping the input $\left(x_{1}, \ldots, x_{s}\right)$ to the output state $W_{x_{1} \ldots x_{s}}$. Without loss of generality we may assume that $\mathfrak{Y}=\mathfrak{L}(\mathcal{H})$ is the full operator algebra of the finite dimensional Hilbert space $\mathcal{H}$, and we shall assume that the $W_{x_{1} \ldots x_{s}}$ are density operators on $\mathcal{H} \cdot 1$

However, to express the theory in this algebraic manner has its merits, as we shall see:

The output state (generally mixed) is accessed by several receicers. These are represented by commuting *subalgebras $\mathfrak{Y}_{j}(j=1, \ldots, r)$ of $\mathfrak{Y}$ : the meaning is that receiver $j$ may use any measurement (POVM) whose elements belong to $\mathfrak{Y}_{j}$. The commutativity ensures that these measurements can be performed together. The typical case

\footnotetext{
${ }^{1}$ In the general case we may make use of the fact that the states of $\mathfrak{Y}$ are uniquely described by density operators inside $\mathfrak{Y}$.
} 
of this situation is that $\mathfrak{Y}=\mathfrak{Y}_{1} \otimes \cdots \otimes \mathfrak{Y}_{r}$, where we identify $\mathfrak{Y}_{j}$ with the subalgebra $\mathbb{1}^{\otimes(j-1)} \otimes \mathfrak{Y}_{j} \otimes \mathbb{1}^{\otimes(r-j)}$ of $\mathfrak{Y}$. By linear extension we may view $W$ as a completely positive, trace preserving map from $\mathfrak{X}_{1} \otimes \cdots \otimes \mathfrak{X}_{s}$ to $\mathfrak{Y}$, where $\mathfrak{X}_{i}=\mathbb{C} \mathcal{X}_{i}$ is the commutative algebra of $\mathbb{C}$-valued functions on $\mathcal{X}_{i}$, whose elements we identify with their indicator functions. [f If all the $W_{x_{1} \ldots x_{s}}$ commute with each other (hence have a common diagonalization) the channel is called quasi-classical, and classical if $\mathfrak{Y}$ is a commutative algebra.

It should be stressed that all this can be embedded into standard quantum theory by identifying all algebras in question with operator algebras in some sufficiently large Hilbert space, e.g. a commutative algebra with a set of diagonal matrices. To get more familiar with this formalism the reader might consult a book like [12].

For fixed a priori distributions define the channel state

$$
\gamma=\sum_{\forall i x_{i} \in \mathcal{X}_{i}} P_{1}\left(x_{1}\right) \cdots P_{s}\left(x_{s}\right) x_{1} \otimes \cdots \otimes x_{s} \otimes W_{x_{1} \ldots x_{s}}
$$

on $\mathfrak{X}_{1} \otimes \cdots \otimes \mathfrak{X}_{s} \otimes \mathfrak{Y}$. This serves as the quantum analogue of the joint distribution of the random variables representing input and output letters in the classical case. It may be interpreted as the joint state of the system after the channel usage, where the senders kept a record of their individual letters (this is possible because they input classical information, reflected in the classical nature of their systems).

Note the 1-1-correspondence between states $\gamma$ on $\mathfrak{X}_{1} \otimes$ $\cdots \otimes \mathfrak{X}_{s} \otimes \mathfrak{Y}$ and pairs $(P, W)$ of channels $W$ and probability distributions $P$ on $\mathcal{X}_{1} \times \cdots \times \mathcal{X}_{s}$. This is a feature of our model, which relies on the commutativity of the $\mathfrak{X}_{i}$ (compare [13] for the difficulties encountered in more general situations).

We will employ block coding on the discrete memoryless channel generated by $W$ : for sequences $x_{1}^{n}, \ldots, x_{s}^{n}, x_{i}^{n}=$ $x_{i 1} \ldots x_{i n} \in \mathcal{X}_{i}^{n}$, the $n$-block channel

$$
W^{n}: \mathcal{X}_{1}^{n} \times \cdots \times \mathcal{X}_{s}^{n} \rightarrow \mathfrak{S}\left(\mathfrak{Y}^{\otimes n}\right)
$$

is defined by

$$
W_{x_{1}^{n} \ldots x_{s}^{n}}^{n}=W_{x_{11} x_{21} \ldots x_{s 1}} \otimes W_{x_{12} x_{22} \ldots x_{s 2}} \otimes \cdots \otimes W_{x_{1 n} x_{2 n} \ldots x_{s n}} .
$$

We now introduce some notation to describe the channel as seen by a subset of senders, while the others enter only stochastically:

For a $J \subset[s]=\{1, \ldots, s\}$ denote $P_{J}=\bigotimes_{i \in J} P_{i}$, i.e. $P_{J}\left(x_{i} \mid i \in J\right)=\prod_{i \in J} P_{i}\left(x_{i}\right)$, and $\mathcal{X}(J)=\prod_{i \in J} \mathcal{X}_{i}$ (similarly $\left.\mathfrak{X}(J)=\bigotimes_{i \in J} \mathfrak{X}_{i}\right)$.

Define the reduced channel $P_{J^{c}} W: \mathcal{X}(J) \rightarrow \mathfrak{S}(\mathfrak{Y})$ by

$$
\left(P_{J^{c}} W\right)_{\left(x_{i} \mid i \in J\right)}=\sum_{\forall i \in J^{c}: x_{i} \in \mathcal{X}_{i}} P_{J^{c}}\left(x_{i} \mid i \in J^{c}\right) W_{x_{1} \ldots x_{s}} .
$$

\footnotetext{
${ }^{2}$ Since all algebras here are finite dimensional we do not care about the topological distictions necessary in general, between linear spaces and their duals, between maps and their adjoints.
}

(Here $J^{c}$ denotes the complement $[s] \backslash J$ of $J$ in $[s]$ ). Note that

$$
\operatorname{Tr}_{\mathfrak{X}\left(J^{c}\right)} \gamma=\sum_{\forall i \in J: x_{i} \in \mathcal{X}_{i}} P_{J}\left(x_{i} \mid i \in J\right) \bigotimes_{i \in J} x_{i} \otimes\left(P_{J^{c}} W\right)_{\left(x_{i} \mid i \in J\right)} .
$$

Transmission is now by using codes on $n$-blocks:

An $n$-block-code is a collection $\left(f_{1}, \ldots, f_{s}, D_{1}, \ldots, D_{r}\right)$ of maps $f_{i}: \mathcal{M}_{i} \rightarrow \mathcal{X}_{i}^{n}$ (where $\mathcal{M}_{i}$ is the set of messages of sender $i$ ) and decoding observables (POVMs) $D_{j} \subset \mathfrak{Y}_{j}^{\otimes n}$, indexed by $\mathcal{M}_{1} \times \cdots \times \mathcal{M}_{s}$, i.e.:

$$
D_{j}=\left\{D_{j \mu} \in \mathfrak{Y}_{j}: \mu \in \mathcal{M}_{1} \times \cdots \times \mathcal{M}_{s}\right\},
$$

such that

$$
D_{j \mu} \geq 0, \quad \sum_{\mu} D_{j \mu}=\mathbb{1} .
$$

There are $r$ (average) error probabilities of the code, the probability that the receiver $j$ guesses incorrectly any one of the sent words, taken over the uniform distribution on the message sets:

$$
\begin{aligned}
& \bar{e}_{j}\left(f_{1}, \ldots, f_{s}, D_{j}\right)= \\
& 1-\frac{1}{\left|\mathcal{M}_{1}\right| \cdots\left|\mathcal{M}_{s}\right|} \sum_{\forall i: m_{i} \in \mathcal{M}_{i}} \operatorname{Tr}\left(W_{f\left(m_{1}\right) \ldots f\left(m_{s}\right)}^{n} D_{j, m_{1} \ldots m_{s}}\right) .
\end{aligned}
$$

We call $\left(f_{1}, \ldots, f_{s}, D_{1}, \ldots, D_{r}\right)$ an $(n, \bar{\epsilon})$-code if all error probabilities $\bar{e}_{j}\left(f_{1}, \ldots, f_{s}, D_{j}\right)$ do not exceed $\bar{\epsilon}$.

The rates of the code are the $R_{i}=\frac{1}{n} \log \left|\mathcal{M}_{i}\right|$. A tuple $\left(R_{1}, \ldots, R_{s}\right)$ is said to be achievable, if for any $\bar{\epsilon}, \delta>0$ there exists for any large enough $n$ an $(n, \bar{\epsilon})$-code with $i_{-}$ th rate at least $R_{i}-\delta$. The set of all achievable tuples (which is clearly closed) is called the capacity region of the channel, and to determine this region is the problem to be addressed here.

Some observations should be made: first, the capacity region is convex, by the time sharing principle: let $\left(R_{1}, \ldots, R_{s}\right)$ and $\left(R_{1}^{\prime}, \ldots, R_{s}^{\prime}\right)$ be rate tuples of $m$ - and $n$-block codes, respectively, with error probability $\bar{\epsilon}$ each. By concatenating the codewords to $(m+n)$-blocks, and tensoring the corresponding decoding observables, we get an $(m+n)$-block code with error probability at most $2 \bar{\epsilon}$, and with rates $\frac{m}{m+n} R_{i}+\frac{n}{m+n} R_{i}^{\prime}$.

Second, note that in the multi-user situation not a single number describes the performance of the channel (as with capacity in the single-sender case). Instead, only with given behaviour of the other senders the channel gets a specific capacity for a particular sender. Intuitively, this is because the others' (unknown!) actions may be seen as additional noise (a phenomenon known as "interference" in classical multi-user channels).

\section{Information Quantities in Quantum Systems}

In this section we introduce some notation in which we express our results. From [17, appendix A, we use the definitions of various information quantities for observables and $*$-subalgebras, which we review for the sake of selfcontainedness: 
Let $\mathfrak{A}$ be a $\mathrm{C}^{*}$-algebra, and $\rho$ a state on it. For a $*-$ subalgebra $\mathfrak{B}$ we want to define the entropy of $\rho$ with respect to this subalgebra (we shall stress the dependence on $\mathfrak{B}$, as $\rho$ is supposed to be fixed). To this end let us consider the restriction $\sigma=\left.\rho\right|_{\mathfrak{B}}$ of $\rho$ to $\mathfrak{B}$, and define

$$
H(\mathfrak{B})=H_{\rho}(\mathfrak{B})=-\operatorname{Tr}(\sigma \log \sigma) .
$$

Here $\operatorname{Tr}$ is the unique trace on $\mathfrak{B}$ (i.e. a positive $\mathbb{C}$-linear functional on $\mathfrak{B}$, with the properties $\operatorname{Tr} A B=\operatorname{Tr} B A$ and $\operatorname{Tr} A^{*}=\overline{\operatorname{Tr} A}$ ), that assigns 1 to all minimal idempotents of $\mathfrak{B}$. An important example is the usual trace of $\mathfrak{L}(\mathcal{H})$, in which case the formula gives the familiar von Neumann entropy of the state.

Motivated by identities for classical Shannon entropy we may now define, for (elementwise) commuting *subalgebras $\mathfrak{B}$ and $\mathfrak{C}$ : the conditional entropy

$$
H(\mathfrak{B} \mid \mathfrak{C})=H(\mathfrak{B} \mathfrak{C})-H(\mathfrak{C}),
$$

and the mutual information

$$
\begin{aligned}
I(\mathfrak{B} \wedge \mathfrak{C}) & =H(\mathfrak{B})+H(\mathfrak{C})-H(\mathfrak{B C}) \\
& =H(\mathfrak{B})-H(\mathfrak{B} \mid \mathfrak{C}) .
\end{aligned}
$$

The condition that the algebras commute is crucial here: it ensures that all observables in $\mathfrak{B}$ are coexistent with all observables in $\mathfrak{C}$, and also, that the product $\mathfrak{B C}$ is indeed the algebra generated by $\mathfrak{B}$ and $\mathfrak{C}$. Of course, these definitions are only formally derived from well known classical formulas, and there is no reason to expect that they are meaningful (which indeed they are only to a limited degree: see the discussions in [4], and in [1]]). Anyhow, for our purposes they make sufficient sense.

If $\mathfrak{D}$ is a third $*$-subalgebra, commuting with both $\mathfrak{B}$ and $\mathfrak{C}$, we may finally define the conditional mutual information

$$
\begin{aligned}
I(\mathfrak{B} \wedge \mathfrak{C} \mid \mathfrak{D}) & =H(\mathfrak{B} \mid \mathfrak{D})+H(\mathfrak{C} \mid \mathfrak{D})-H(\mathfrak{B} \mathfrak{C} \mid \mathfrak{D}) \\
& =H(\mathfrak{B D})+H(\mathfrak{C} \mathfrak{D})-H(\mathfrak{B C} \mathfrak{D})-H(\mathfrak{D}) .
\end{aligned}
$$

We note, that the conditional mutual information is positive, by the strong subadditivity of von Neumann entropy (see [17], theorem A.9).

In all the above expressions we supressed the dependence on the underlying state $\rho$. In cases of possible ambiguity it is added as a subscript.

With these definitions we have the (easily checked) identities for the system introduced in section II, with the channel state $\gamma$ :

$$
\begin{aligned}
I\left(\mathfrak{X}(J) \wedge \mathfrak{Y} \mid \mathfrak{X}\left(J^{c}\right)\right) & =I\left(\mathfrak{X}(J) \wedge \mathfrak{Y} \mathfrak{X}\left(J^{c}\right)\right) \\
& =H\left(\mathfrak{Y} \mid \mathfrak{X}\left(J^{c}\right)\right)-H\left(\mathfrak{Y} \mid \mathfrak{X}_{1} \cdots \mathfrak{X}_{s}\right) \\
& =H\left(P_{J} W \mid P_{J^{c}}\right)-H\left(W \mid P_{[s]}\right),
\end{aligned}
$$

where in the last line an alternative notation is used:

For a channel $V: \mathcal{A} \rightarrow \mathfrak{S}(\mathfrak{Z})$ and a probability distribution $Q$ on $\mathcal{A}$ let

$$
H(V \mid Q)=\sum_{a \in \mathcal{A}} Q(a) H\left(V_{a}\right)
$$

with the von Neumann entropy $H$ : so this is the familiar writing of a conditional as an average of entropies.

There are a number of important relations between all these quantities, of which we shall make use of two:

Lemma 1: Let $V_{k}: \mathcal{A}_{k} \rightarrow \mathfrak{S}\left(\mathfrak{Z}_{k}\right)(k=1,2)$ be two channels, and $Q$ a probability distribution on $\mathcal{A}_{1} \times \mathcal{A}_{2}$. Forming the channel state

$$
\gamma=\sum_{a_{k} \in \mathcal{A}_{k}: k=1,2} Q\left(a_{1}, a_{2}\right) a_{1} \otimes V_{1, a_{1}} \otimes a_{2} \otimes V_{2, a_{2}},
$$

we have the following subadditivity relation:

$$
I\left(\mathbb{C} \mathcal{A}_{1} \mathbb{C} \mathcal{A}_{2} \wedge \mathfrak{Z}_{1} \mathfrak{Z}_{2}\right) \leq I\left(\mathbb{C} \mathcal{A}_{1} \wedge \mathfrak{Z}_{1}\right)+I\left(\mathbb{C} \mathcal{A}_{2} \wedge \mathfrak{Z}_{2}\right)
$$

Proof: This is well known for classical channels, and the proof in our case runs exactly the same. Compare [17, theorem A.17.

Lemma 2 (Fano inequality) Let $\mathfrak{X}, \mathfrak{Y}$ be commuting algebras, and $\mathfrak{X}$ be commutative. For a state $\rho$ on $\mathfrak{X Y}$ consider POVMs $X \subset \mathfrak{X}, Y \subset \mathfrak{Y}$, running over the same index set.

Then the probability of the event " $X \neq Y$ ", i.e.

$$
P_{e}=1-\sum_{j} \operatorname{Tr}\left(\rho X_{j} Y_{j}\right)
$$

satisfies

$$
H(\mathfrak{X} \mid \mathfrak{Y}) \leq 1+P_{e} \log \operatorname{Tr} \mathbb{1}_{\mathfrak{X}} .
$$

Proof: See 17], corollary A.25. Observe that the statement of the lemma is a way of expressing the Holevo bound 7 .

\section{UPPER CAPACITY Bounds}

The following theorem (which we call the weak converse because of theorem 9 and note 4 ) was, in the case $r=1$ and $s=2$, stated in $[3]$.

Theorem 3 (Weak converse) The capacity region of the quantum multiway channel is contained in the closure of all nonnegative $\left(R_{1}, \ldots, R_{s}\right)$ satisfying for all $J \subset[s]$ and $j \in[r]$

$$
R(J)=\sum_{i \in J} R_{i} \leq \sum_{u} q_{u} I_{\gamma_{u}}\left(\mathfrak{X}(J) \wedge \mathfrak{Y}_{j} \mid \mathfrak{X}\left(J^{c}\right)\right)
$$

for channel states $\gamma_{u}$ and $q_{u} \geq 0, \sum_{u} q_{u}=1$.

Proof: $\quad$ Let $\left(f_{1}, \ldots, f_{s}, D_{1}, \ldots, D_{r}\right)$ be any $(n, \bar{\epsilon})-$ code with rate tuple $\left(R_{1}, \ldots, R_{s}\right)$. Then the uniform distribution on the codewords induces a channel state $\gamma$ on $\left(\mathfrak{X}_{1} \cdots \mathfrak{X}_{s} \mathfrak{Y}\right)^{\otimes n}$ :

$\gamma=\frac{1}{\left|\mathcal{M}_{1}\right| \cdots\left|\mathcal{M}_{s}\right|} \sum_{\forall i:} \bigotimes_{m_{i} \in \mathcal{M}_{i}} \oint_{i} f_{i}\left(m_{i}\right) \otimes W_{f_{1}\left(m_{1}\right) \ldots f_{s}\left(m_{s}\right)}^{n}$

Its restriction to the $u$-th copy in this tensor power will be denoted $\gamma_{u}$. Let $j \in[r], J \subset[s]$ : by Fano inequality (lemma 2) we have

$$
H\left(\mathfrak{X}^{\otimes n}(J) \mid \mathfrak{Y}_{j}^{\otimes n} \mathfrak{X}^{\otimes n}\left(J^{c}\right)\right) \leq 1+\bar{\epsilon} \cdot n R(J) .
$$


With

$$
\begin{aligned}
H\left(\mathfrak{X}^{\otimes n}(J) \mid\right. & \left.\mathfrak{Y}_{j}^{\otimes n} \mathfrak{X}^{\otimes n}\left(J^{c}\right)\right) \\
& =H\left(\mathfrak{X}^{\otimes n}(J)\right)-I\left(\mathfrak{X}^{\otimes n}(J) \wedge \mathfrak{Y}_{j}^{\otimes n} \mathfrak{X}^{\otimes n}\left(J^{c}\right)\right) \\
& =n R(J)-I\left(\mathfrak{X}^{\otimes n}(J) \wedge \mathfrak{Y}_{j}^{\otimes n} \mathfrak{X}^{\otimes n}\left(J^{c}\right)\right)
\end{aligned}
$$

we conclude now

$$
\begin{aligned}
(1-\bar{\epsilon}) R(J) & \leq \frac{1}{n}+\frac{1}{n} I_{\gamma}\left(\mathfrak{X}^{\otimes n}(J) \wedge \mathfrak{Y}_{j}^{\otimes n} \mathfrak{X}^{\otimes n}\left(J^{c}\right)\right) \\
& \leq \frac{1}{n}+\frac{1}{n} \sum_{u=1}^{n} I_{\gamma_{u}}\left(\mathfrak{X}(J) \wedge \mathfrak{Y}_{j} \mathfrak{X}\left(J^{c}\right)\right),
\end{aligned}
$$

using lemma 1 (subadditivity of mutual information).

Remark 4: For classical channels the region described in the theorem is the exact capacity region (i.e. all the rates there are achievable), as was first proved by Ahlswede [1], [2]. This fact is our reason to call it the weak converse, as it describes the best outer bounds of $(n, \bar{\epsilon})$-code rates for $n \rightarrow \infty$ and $\bar{\epsilon} \rightarrow 0$.

To prove that for multiple access channels $(r=1)$ this holds, too, is the object of the rest of the paper, though we conjecture it to be true in general.

Remark 5: The numerical computation of the above regions is not yet possible from the given description: we need a bound on the number of different single-letter channel states one has to consider in the convex combinations. For the multiple access channel $(r=1)$ this is easy: by Caratheodory's theorem $s$ will suffice. For general $r$ it is possible to show that $r\left(2^{s}-1\right)$ are sufficient (cf. [5]).

\section{Measurement Error and Disturbance}

In this section a central result is proved that essentially states that if a POVM serves to indentify the states of an ensemble with high probability, then it may be implemented as an operation that disturbes the ensemble states very little.

Lemma 6: Let $\rho$ be a state, and $X$ a positive operator with $X \leq \mathbb{1}$ and $1-\operatorname{Tr}(\rho X) \leq \epsilon<1$. Then

$$
\|\rho-\sqrt{X} \rho \sqrt{X}\|_{1} \leq \sqrt{8 \epsilon},
$$

with the trace norm $\|X\|_{1}=\operatorname{Tr}|X|$.

Proof: See [18], lemma V.9.

Lemma 7 (Tender measurement) Let $\rho_{a}(a \in \mathcal{A})$ be a set of states on $\mathfrak{A}$, and $D$ an observable indexed by $\mathcal{B}$. Let further $\varphi: \mathcal{A} \rightarrow \mathcal{B}$ be any map and $\epsilon>0$ such that

$$
\forall a \in \mathcal{A} \quad 1-\operatorname{Tr}\left(\rho_{a} D_{\varphi(a)}\right) \leq \epsilon
$$

(i.e. the observable recognizes $\varphi(a)$ from $\rho_{a}$ with maximal error probability $\epsilon)$. Then the quantum operation $\delta: \mathfrak{S}(\mathfrak{A}) \rightarrow \mathfrak{S}(\mathfrak{A})$ defined by

$$
\delta: \rho \mapsto \sum_{b \in \mathcal{B}} \sqrt{D_{b}} \rho \sqrt{D_{b}}
$$

disturbes the states $\rho_{a}$ only a little:

$$
\forall a \in \mathcal{A} \quad\left\|\rho_{a}-\delta\left(\rho_{a}\right)\right\|_{1} \leq \sqrt{8 \epsilon}+\epsilon .
$$

The quantum operation $\Delta: \mathfrak{S}(\mathfrak{A}) \rightarrow \mathfrak{S}(\mathbb{C B} \otimes \mathfrak{A})$ with

$$
\Delta: \rho \mapsto \sum_{b \in \mathcal{B}} b \otimes \sqrt{D_{b}} \rho \sqrt{D_{b}}
$$

has the property that

$$
\forall a \in \mathcal{A} \quad\left\|\varphi(a) \otimes \rho_{a}-\Delta\left(\rho_{a}\right)\right\|_{1} \leq \sqrt{8 \epsilon}+\epsilon .
$$

Proof: It suffices to prove the second statement since the first inequality is obtained from it by a partial trace which does not increase $\|\cdot\|_{1}$ :

$$
\begin{aligned}
&\left\|\varphi(a) \otimes \rho_{a}-\Delta\left(\rho_{a}\right)\right\|_{1} \leq\left\|\rho_{a}-\sqrt{D_{\varphi(a)}} \rho_{a} \sqrt{D_{\varphi(a)}}\right\|_{1} \\
&+\sum_{b \neq \varphi(a)}\left\|\sqrt{D_{b}} \rho_{a} \sqrt{D_{b}}\right\|_{1} \\
&=\left\|\rho_{a}-\sqrt{D_{\varphi(a)}} \rho_{a} \sqrt{D_{\varphi(a)}}\right\|_{1} \\
&+\sum_{b \neq \varphi(a)} \operatorname{Tr}\left(\rho_{a} D_{b}\right) \\
& \leq \sqrt{8\left(1-\operatorname{Tr}\left(\rho_{a} D_{\varphi(a)}\right)\right)} \\
&+\left(1-\operatorname{Tr}\left(\rho_{a} D_{\varphi(a)}\right)\right) \\
& \leq \sqrt{8 \epsilon}+\epsilon
\end{aligned}
$$

using triangle inequality and lemma 6 .

Lemma 8 (Average version) With the same definitions as in lemma 7 , only replacing equation (1) with

$$
\sum_{a \in \mathcal{A}} P(a)\left(1-\operatorname{Tr}\left(\rho_{a} D_{\varphi(a)}\right)\right) \leq \bar{\epsilon}
$$

for a probability distribution $P$ on $\mathcal{A}$, we have

$$
\sum_{a \in \mathcal{A}} P(a)\left\|\rho_{a}-\delta\left(\rho_{a}\right)\right\|_{1} \leq \sqrt{8 \bar{\epsilon}}+\bar{\epsilon}
$$

and

$$
\sum_{a \in \mathcal{A}} P(a)\left\|\varphi(a) \otimes \rho_{a}-\Delta\left(\rho_{a}\right)\right\|_{1} \leq \sqrt{8 \bar{\epsilon}}+\bar{\epsilon} .
$$

Proof: Again, we have only to prove the second statement. Introducing $\epsilon_{a}=1-\operatorname{Tr}\left(\rho_{a} D_{\varphi(a)}\right)$ we have, like in the previous proof,

$$
\left\|\varphi(a) \otimes \rho_{a}-\Delta\left(\rho_{a}\right)\right\|_{1} \leq \sqrt{8 \epsilon_{a}}+\epsilon_{a} .
$$

Forming the average of the left hand side under the distribution $P$, and using concavity of $\sqrt{8 x}+x$ the assertion follows.

\section{Quantum Multiple Access Channel: Coding}

Throughout this section we will assume $r=1$ and else notation as before.

Theorem 9: Let $R_{1}, \ldots, R_{s}$ be nonnegative, satisfying for some a priori distributions $P_{i}$ on the $\mathcal{X}_{i}$ the constraints

$$
\forall J \subset[s] \quad \sum_{i \in J} R_{i} \leq I\left(\mathfrak{X}(J) \wedge \mathfrak{Y} \mid \mathfrak{X}\left(J^{c}\right)\right) .
$$


Then for every $\bar{\epsilon}, \delta>0$ and all sufficiently large $n$ there are $(n, \bar{\epsilon})$-codes with rates $\frac{1}{n} \log \left|\mathcal{M}_{i}\right| \geq R_{i}-\delta$.

Proof: It is sufficient by the time sharing principle to prove the assertion only for the upper extremal points of the region described, and by symmetry we may assume (for $i=1, \ldots, s$ ) that

$$
\begin{aligned}
R_{i} & =I\left(\mathfrak{X}_{i} \wedge \mathfrak{Y} \mathfrak{X}_{1} \cdots \mathfrak{X}_{i-1}\right) \\
& =H\left(\mathfrak{Y} \mid \mathfrak{X}_{1} \cdots \mathfrak{X}_{i-1}\right)-H\left(\mathfrak{Y} \mid \mathfrak{X}_{1} \cdots \mathfrak{X}_{i}\right) \\
& =H\left(P_{\{>i-1\}} W \mid P_{\{\leq i-1\}}\right)-H\left(P_{\{>i\}} W \mid P_{\{\leq i\}}\right) .
\end{aligned}
$$

That these are indeed the upper extreme points is proved in the appendix.

The idea of the following construction is to first decode the message $m_{1}$ from sender 1 , using only the incoming signal. Then decode the message $m_{2}$ from sender 2, using $m_{1}$ and the incoming signal (which is almost undisturbed by the tender measurement lemma). Iterate, until you decode message $m_{s}$ from sender $s$, using $m_{1}, \ldots, m_{s-1}$ and the still almost unchanged incoming signal.

Let $\epsilon, \delta>0$, and consider $s$ families of codewords $\mathcal{C}_{i} \subset$ $\mathcal{X}_{i}^{n}$ of size $L_{i}=\left|\mathcal{C}_{i}\right|=\left\lceil 2^{n\left(R_{i}-\delta\right)}\right\rceil$, drawn independently from $\mathcal{X}_{i}^{n}$ according to the a priori distribution $P_{i}^{\otimes n}$.

Fix $i$ for the moment and define the following channel: for $x_{i}^{n} \in \mathcal{X}_{i}^{n}$

$$
\begin{aligned}
& \rho^{(i)}: x_{i}^{n} \mapsto \rho_{x_{i}^{n}}^{(i)}= \\
& \frac{1}{L_{1} \cdots L_{i-1} L_{i+1} \cdots L_{s}} \sum_{\forall j \neq i: c_{j}^{n} \in \mathcal{C}_{j}}\left(\bigotimes_{j<i} c_{j}^{n} \otimes W_{c_{1}^{n} \ldots x_{i}^{n} \ldots c_{s}^{n}}^{n}\right)
\end{aligned}
$$

(we denote these word states by $\rho^{(i)}$, in contrast to the letter states $W$ ). Note that this is the channel belonging to the channel state $\gamma$ from the proof of theorem 3 , reduced to $\left(\mathfrak{X}_{1} \ldots \mathfrak{X}_{i-1} \mathfrak{Y}\right)^{\otimes n}$.

The average of $\rho_{x_{i}^{n}}^{(i)}$ over the choice of $\mathcal{C}_{1}, \ldots, \mathcal{C}_{i-1}$, $\mathcal{C}_{i+1}, \ldots, \mathcal{C}_{s}$ is indeed a product state:

$$
\begin{aligned}
& \left\langle\rho_{x_{i}^{n}}^{(i)}\right\rangle_{\mathcal{C}_{i}: i \neq j}=\sum_{\forall j \neq i: x_{j}^{n} \in \mathcal{X}_{j}^{n}} P_{\{\neq i\}}\left(x_{j}^{n} \mid j \neq i\right) \bigotimes_{j<i} x_{j}^{n} \otimes W_{x_{1}^{n} \ldots x_{s}^{n}}^{n} \\
& \quad=\sum_{\forall j<i: x_{j}^{n} \in \mathcal{X}_{j}^{n}} P_{\{<i\}}\left(x_{j}^{n} \mid j<i\right) \bigotimes_{j<i} x_{j}^{n} \otimes\left(P_{\{>i\}} W\right)_{x_{1}^{n} \ldots x_{i}^{n}}^{n} \\
& \quad=V_{x_{i}^{n}}^{n},
\end{aligned}
$$

with

$$
V_{x_{i}}=\sum_{\forall j<i: x_{j} \in \mathcal{X}_{j}} P_{\{<i\}}\left(x_{j} \mid j<i\right) \bigotimes_{j<i} x_{j} \otimes\left(P_{\{>i\}} W\right)_{x_{1} \ldots x_{i}} .
$$

In [9] and [15] a construction of a decoding observable for the channel $V$ and set $\mathcal{C}_{i}$ of codewords is described, ${ }^{3}$ which has the property that it's average error probability

$$
\bar{e}_{V^{n}}\left(\mathcal{C}_{i}, D_{i}\right)=1-\frac{1}{\left|\mathcal{C}_{i}\right|} \sum_{c_{i}^{n} \in \mathcal{C}_{i}} \operatorname{Tr}\left(V_{c_{i}^{n}}^{n} D_{i, c_{i}^{n}}\right),
$$

\footnotetext{
${ }^{3}$ Observe that in this way $D_{i}$ will be independent from the other codes and their decoding observables!
}

averaged over the choice of $\mathcal{C}_{i}$, is at most $\epsilon / s$ for large enough $n$ :

$$
\left\langle\bar{e}_{V^{n}}\left(\mathcal{C}_{i}, D_{i}\right)\right\rangle_{\mathcal{C}_{i}} \leq \epsilon / s
$$

(where we identified the set of messages with $\mathcal{C}_{i}$ ). This is because $I\left(P_{i} ; V\right)=I\left(\mathfrak{X}_{i} \wedge \mathfrak{Y} \mathfrak{X}_{1} \cdots \mathfrak{X}_{i-1}\right)=R_{i}$. (Recall the approach of [9] and [15]: a random code - drawn according to $P_{i}^{\otimes n}$ - is chosen with rate slightly below $I\left(P_{i} ; V\right)$, and a decoding POVM constructed which forces the expected average error probability small. Then it is concluded that a code with this small error probability actually exists).

We note that by the construction from [9] and [15] it is assured that

$$
D_{i, c_{i}^{n}} \in\left(\mathfrak{X}_{1} \cdots \mathfrak{X}_{i-1} \mathfrak{Y}\right)^{\otimes n},
$$

for all $c_{i}^{n} \in \mathcal{C}_{i}$. It is easlity seen that we may assume this w.l.o.g., for wherever the $D_{i}$ comes from: the $V_{c_{i}^{n}}^{n}$ are density operators on some Hilber space $\mathcal{H}$, such that

$$
V_{c_{i}^{n}}^{n} \in\left(\mathfrak{X}_{1} \cdots \mathfrak{X}_{i-1} \mathfrak{Y}\right)^{\otimes n} \subset \mathfrak{L}(\mathcal{H}) .
$$

Denoting this subset embedding by $E$, we have

$$
\operatorname{Tr}\left(V_{c_{i}^{n}}^{n} D_{i, c_{i}^{n}}\right)=\operatorname{Tr}\left(E\left(V_{c_{i}^{n}}^{n}\right) D_{i, c_{i}^{n}}\right)=\operatorname{Tr}\left(V_{c_{i}^{n}}^{n} E^{*}\left(D_{i, c_{i}^{n}}\right)\right),
$$

with the adjoint map $E^{*}$. I.e. the decoding error probabilities do not change if we replace the $D_{i, c_{i}^{n}}$ by $E^{*}\left(D_{i, c_{i}^{n}}\right) \in$ $\left(\mathfrak{X}_{1} \cdots \mathfrak{X}_{i-1} \mathfrak{Y}\right)^{\otimes n}$.

Now we confront the decoder $D_{i}$ with the signal $\rho$, obtaining an average error probability

$$
\bar{e}_{i}=\bar{e}_{\rho^{(i)}}\left(\mathcal{C}_{i}, D_{i}\right)=1-\frac{1}{L_{i}} \sum_{c_{i}^{n} \in \mathcal{C}_{i}} \operatorname{Tr}\left(\rho_{c_{i}^{n}} D_{i, c_{i}^{n}}\right) .
$$

Obviously the code average over choice of $\mathcal{C}_{j}, j \neq i$ is exactly $\bar{e}_{V^{n}}\left(\mathcal{C}_{i}, D_{i}\right)$, so by the previous argument

$$
\left\langle\bar{e}_{1}+\ldots+\bar{e}_{s}\right\rangle_{\mathcal{C}_{1} \ldots \mathcal{C}_{s}} \leq \epsilon .
$$

This means that there actually exist codebooks $\mathcal{C}_{1}, \ldots, \mathcal{C}_{s}$ with all the $\bar{e}_{i} \leq \epsilon$. Now consider the corresponding operations $\Delta_{i}$ of the observables $D_{i}$, from lemma 7 . By the average tender measurement lemma 8 we find for each $i$ :

$$
\begin{aligned}
& \frac{1}{L_{1} \cdots L_{s}} \sum_{\forall j: c_{j}^{n} \in \mathcal{C}_{j}}\left\|\bigotimes_{j \leq i} c_{j}^{n} \otimes W_{c_{1}^{n} \ldots c_{s}^{n}}^{n}-\Delta_{i}\left(\bigotimes_{j<i} c_{j}^{n} \otimes W_{c_{1}^{n} \ldots c_{s}^{n}}^{n}\right)\right\|_{1} \\
& \leq \sqrt{8 \epsilon}+\epsilon .
\end{aligned}
$$

Their concatenation $\Delta=\Delta_{s} \circ \cdots \circ \Delta_{1}$ will be the decoder process: it satisfies

$$
\begin{aligned}
& \frac{1}{L_{1} \cdots L_{s}} \sum_{\forall j: c_{j}^{n} \in \mathcal{C}_{j}}\left\|\bigotimes_{j=1}^{s} c_{j}^{n} \otimes W_{c_{1}^{n} \ldots c_{s}^{n}}^{n}-\Delta\left(W_{c_{1}^{n} \ldots c_{s}^{n}}^{n}\right)\right\|_{1} \\
& \quad \leq s(\sqrt{8 \epsilon}+\epsilon),
\end{aligned}
$$

since each step introduces a deviation of at most $\sqrt{8 \epsilon}+\epsilon$, and does not increase trace norm. 
Now, discarding the signal itself (i.e tracing out $\mathfrak{Y}^{\otimes n}$ ), we arrive at $\widetilde{\Delta}=\operatorname{Tr}_{\mathfrak{Y} \otimes n} \circ \Delta$, which satisfies

$$
\frac{1}{L_{1} \cdots L_{s}} \sum_{\forall j: c_{j}^{n} \in \mathcal{C}_{j}}\left\|\bigotimes_{j=1}^{s} c_{j}^{n}-\widetilde{\Delta}\left(W_{c_{1}^{n} \ldots c_{s}^{n}}^{n}\right)\right\|_{1} \leq s(\sqrt{8 \epsilon}+\epsilon) .
$$

This means, that $\widetilde{\Delta}$ is a quantum operation with classical outcomes in $\mathcal{C}_{1} \times \cdots \times \mathcal{C}_{s}$ (hence described by a POVM $D$ indexed by this set), which recovers rather accurately $\left(c_{1}^{n}, \ldots, c_{s}^{n}\right)$ from $W_{c_{1}^{n} \ldots c_{s}^{n}}^{n}$, on average.

Now we may combine the (weak) converse theorem 3 and the foregoing direct coding theorem 9 (together with the time sharing principle by which the capacity region is convex) to obtain

Theorem 10: The capacity region of the quantum multiple access channel is the convex closure of all nonnegative $\left(R_{1}, \ldots, R_{s}\right)$ satisfying

$$
\forall J \subset[s] \quad \sum_{i \in J} R_{i} \leq I\left(\mathfrak{X}(J) \wedge \mathfrak{Y} \mid \mathfrak{X}\left(J^{c}\right)\right)
$$

for some input distribution and corresponding channel state.

\section{Quantum-Quantum Multiway Channels}

Allahverdyan and Saakian in [3] actually defined quantum multiway channels in more general form: namely allowing in the definition of section III general $\mathrm{C}^{*}$-algebras $\mathfrak{X}_{i}$ (and not only commutative ones as we did). Each sender $i$ may then use any set of states on $\mathfrak{X}_{i}$ for transmission. Formally, let

$$
\varphi: \mathfrak{S}\left(\mathfrak{X}_{1} \otimes \cdots \otimes \mathfrak{X}_{s}\right) \rightarrow \mathfrak{S}(\mathfrak{Y})
$$

be any completely positive, trace preserving, linear map, i.e. a quantum operation on states. A code for sender $i$ now consists of a map $F_{i}: \mathcal{M}_{i} \rightarrow \mathfrak{S}\left(\mathfrak{X}_{i}^{\otimes n}\right)$, the decoders are still observables in the algebras $\mathfrak{Y}_{j}$. This allows for definition of error probabilities and rates, and hence of the capacity region, the details of which we leave to the reader.

By composition we can view this as a classical-quantum channel on $n$-blocks:

$$
\left(m_{1}, \ldots, m_{s}\right) \mapsto \varphi^{\otimes n}\left(F_{1}\left(m_{1}\right) \otimes \cdots \otimes F_{s}\left(m_{s}\right)\right) .
$$

If we now make the restriction that in coding the senders have to use product states, i.e.

$$
F_{i}\left(m_{i}\right)=F_{i, m_{i}, 1} \otimes \cdots \otimes F_{i, m_{i}, n}
$$

with $F_{i, m_{i}, k} \in \mathfrak{S}\left(\mathfrak{X}_{i}\right)$, we see immediately that the argument from section [V] applies, and gives a general upper bound theorem (with the slight difference that now in the formation of the channel states $\gamma_{u}$ instead of the states $W_{f_{1}\left(m_{1}\right)_{u} \ldots f_{s}\left(m_{s}\right)_{u}}$ there occur the output states $\left.\varphi\left(F_{1, m_{1}, u} \otimes \cdots \otimes F_{s, m_{s}, u}\right)\right)$.

Conversely: those input states and a distribution on them being chosen we can apply the coding theorem 9 to see that these bounds can be approached - at least for the multiple access channel.

We have thus obtained the capacity region $\mathbf{R}^{(1)}$ in the 1-separable case, i.e. for input states that are separable (it is easy to see that best performance in this case is for product states). Of course we could allow the sender to use blocks of states which entangle at most $\nu$ systems, getting a possibly larger region $\mathbf{R}^{(\nu)}$. This is because now the subadditivity of the mutual information, used in the upper bound in section IV, does not hold any longer. We leave open the question of the ultimate capacity region $\mathbf{R}=$ $\overline{\bigcup_{\nu} \mathbf{R}^{(\nu)}}$ (which even for the case $s=r=1$ is unsolved): whether it be equal or strictly larger than $\mathbf{R}^{(1)}$.

\section{CONCLUSION}

We introduced quantum multiway channel and bounded its capacity region. In the case of the quantum multiple access channel this led to a complete characterization of the capacity region, formally identical to the result in the classical case. This confirms the validity of the principle that in the theory of classical information transmission over a quantum channel capacities are obtained from the classical formulas by replacing Shannon entropy by von Neumann entropy.

The next step is of course to prove the direct coding theorem in the case of several receivers. It seems that for this a refinement of the methods used here or even a different approach is needed: namely, it is known that it is not sufficient to achieve rate tuples of the form used in the proof of theorem 9. Hence the method of sequentially decoding the messages of the different senders has to be abandoned.

A note on the literature: Since the findings of the present paper (published at that time only as an e-print (quant-ph/9807019)) an article by Huang, Zhang, and Hou 10 on quantum multiple access channels has appeared, where the 2 -sender pure state case of a multiple access channel is treated.

In that work a reference to the above statement was made concerning the wish for a more direct proof of the achievability of the region described in the coding theorem 10. The authors propose to supply such a proof. However, they too employ the present paper's method to show achievability of the "corners" (compare theorem 9), and then invoke the time-sharing principle. As was pointed out here this method is not adapted to prove the theorem for multiple receivers: it is still an open problem to find a proof that directly allows to achieve every point of the capacity region. We most probably know how to perform the random code selection, but the analog of the maximum likelihood decoder necessary to decode all messages simultaneously still evades us (for detail how this strategy works in the classical case, see [2]).

\section{ACKNOWLEDGEMENTS}

The author wishes to thank Peter Löber for discussions on the subject, and Prof. Rudolf Ahlswede for comments on the proof of the classical theorem. 


\section{APPENDIX}

\section{EXTREMAL POINTS}

We shall prove: any upper extremal point of the region of non-negative tuples $\left(R_{1}, \ldots, R_{s}\right)$ which satisfy for all $J \subset[s]$

$$
R(J) \leq I\left(\mathfrak{X}(J) \wedge \mathfrak{Y} \mathfrak{X}\left(J^{c}\right)\right),
$$

for fixed channel state, is one of the $\left(R_{1}, \ldots, R_{s}\right)$ with

$$
R_{\pi(i)}=I\left(\mathfrak{X}_{\pi(i)} \wedge \mathfrak{Y} \mathfrak{X}_{\pi(1)} \cdots \mathfrak{X}_{\pi(i-1)}\right)
$$

for some permutation $\pi$ of the set $[s]$, and any such tuple belongs to this region.

Let $\left(R_{1}, \ldots, R_{s}\right)$ be an upper extremal point, hence $s$ of the inequalities (for nonempty subsets $K_{1}, \ldots, K_{s}$ of $[s]$ ) are met with equality. We are done if we can prove that these can be chosen to form a strictly increasing chain $\emptyset \neq$ $K_{1} \subset K_{2} \subset \ldots \subset K_{s}=[s]$, w.l.o.g. $K_{i}=\{s, s-1, \ldots, s-$ $i+1\}$, because then

$$
\begin{aligned}
R_{i}= & I\left(\mathfrak{X}_{i} \cdots \mathfrak{X}_{s} \wedge \mathfrak{Y} \mathfrak{X}_{1} \cdots \mathfrak{X}_{i-1}\right) \\
& -I\left(\mathfrak{X}_{i+1} \cdots \mathfrak{X}_{s} \wedge \mathfrak{Y} \mathfrak{X}_{1} \cdots \mathfrak{X}_{i}\right) \\
= & I\left(\mathfrak{X}_{i} \wedge \mathfrak{Y} \mathfrak{X}_{1} \cdots \mathfrak{X}_{i-1}\right) .
\end{aligned}
$$

Choose any one such set, say $K \neq \emptyset$ :

$$
R(K)=I\left(\mathfrak{X}(K) \wedge \mathfrak{Y} \mathfrak{X}\left(K^{c}\right)\right) .
$$

We would like to employ induction to extend this $K$ in both directions to a chain. That we can do so follows from

Lemma 11: If $R_{1}, \ldots, R_{s} \geq 0$ are such that with $\emptyset \neq$ $K \subset[s]: R(K)=I\left(\mathfrak{X}(K) \wedge \mathfrak{Y} \mathfrak{X}\left(K^{c}\right)\right)$ and $(J)$ holds for all $J \subset K$ and for all $K \subset J \subset[s]$. Then $(J)$ holds for all $J \subset[s]$.

Proof: $\quad$ First let $K \subset J \subset[s]$. Then $(J)$, together with $(K)$ met with equality, implies

$$
\begin{aligned}
R(J \backslash K) & \leq H\left(\mathfrak{Y} \mid \mathfrak{X}\left(J^{c}\right)\right)-H\left(\mathfrak{Y} \mid \mathfrak{X}\left(K^{c}\right)\right) \\
& \leq H\left(\mathfrak{Y} \mid \mathfrak{X}\left((J \backslash K)^{c}\right)\right)-H(\mathfrak{Y} \mid \mathfrak{X}([s])) \\
& =I\left(\mathfrak{X}(J \backslash K) \wedge \mathfrak{Y} \mid \mathfrak{X}\left((J \backslash K)^{c}\right)\right),
\end{aligned}
$$

which is equation $(J \backslash K)$. There the second estimate is by strong subadditivity applied to $\mathfrak{A}_{1}=\mathfrak{X}(J \backslash K), \mathfrak{A}_{2}=$ $\mathfrak{Y} \mathfrak{X}\left(J^{c}\right), \mathfrak{A}_{3}=\mathfrak{X}(K)$.

Now let $J \subset[s]$ be arbitrary and write $J=J_{1} \cup J_{2}$ with $J_{1}=J \cap K, J_{2}=J \backslash K$. Then by assumption and the previous

$$
\begin{aligned}
R(J)= & R\left(J_{1}\right)+R\left(J_{2}\right) \\
\leq & H\left(\mathfrak{Y} \mid \mathfrak{X}\left(J_{1}^{c}\right)\right)-H(\mathfrak{Y} \mid \mathfrak{X}([s])) \\
& \quad+H\left(\mathfrak{Y} \mid \mathfrak{X}\left(J_{2}^{c} \cap K^{c}\right)\right)-H\left(\mathfrak{Y} \mid \mathfrak{X}\left(K^{c}\right)\right) \\
= & H\left(\mathfrak{Y} \mathfrak{X}\left(J_{1}^{c}\right)\right)+H\left(\mathfrak{Y} \mathfrak{X}\left(K^{c} \cap J_{2}^{c}\right)\right)-H\left(\mathfrak{Y} \mathfrak{X}\left(K^{c}\right)\right) \\
& \quad-H\left(\mathfrak{X}\left(\left(K \backslash J_{1}\right) \dot{\cup}\left(K^{c} \backslash J_{2}\right)\right)\right)-H(\mathfrak{Y} \mid \mathfrak{X}([s])) \\
\leq & H\left(\mathfrak{Y} \mathfrak{X}\left(J_{1}^{c} \cap J_{2}^{c}\right)\right)-H\left(\mathfrak{X}\left(J_{1}^{c} \cap J_{2}^{c}\right)\right)-H(\mathfrak{Y} \mid \mathfrak{X}([s])) \\
= & H\left(\mathfrak{Y} \mid \mathfrak{X}\left(J_{1}^{c} \cap J_{2}^{c}\right)\right)-H(\mathfrak{Y} \mid \mathfrak{X}([s])) \\
= & I\left(\mathfrak{X}(J) \wedge \mathfrak{Y} \mid \mathfrak{X}\left(J^{c}\right)\right) .
\end{aligned}
$$

There the second estimate is again by strong subadditivity, with $\mathfrak{A}_{1}=\mathfrak{X}\left(J_{2}\right), \mathfrak{A}_{2}=\mathfrak{Y} \mathfrak{X}\left(K^{c} \cap J_{2}^{c}\right), \mathfrak{A}_{3}=\mathfrak{X}\left(K \backslash J_{1}\right)$.

It follows that if $\left(K^{\prime}\right)$ is met with equality for any $K^{\prime}$, also $\left(K^{\prime} \cap K\right)$ and $\left(K^{\prime} \cup K\right)$ are met with equality. It is easily seen that both below and above $K$ there must occur equalities (except already $|K|=1$ or $K=[s]$ ).

It remains to show that $\left(R_{1}, \ldots, R_{s}\right)$ defined by $(\beta)$ belongs to the region. But this is clear by the same argument as above, used top-down: the conjunction of all $(J)$ with $J \subset[s]$ is implied by the conjunction of all with $J \subset[s-1]$, this in turn inductively by all with $J \subset[s-2]$ etc.

\section{REFERENCES}

[1] R. Ahlswede, "Multi-way communication channels", in: Second International Symposium on Information Theory, pp. 2352, Hungarian Academy of Sciences 1971.

[2] R. Ahlswede, "The capacity region of a channel with two senders and two receivers", Ann. Prob., vol. 2, no. 5, pp. 805-814, 1974.

[3] A. E. Allahverdyan, D. B. Saakian, "Accessible information in multi-access quantum channels", in: Quantum computing and quantum communications (Palm Springs, CA, 1998), pp. 276284, LNCS 1509, Springer, Berlin, 1999.

[4] N. Cerf, C. Adami, "Information theory of quantum entanglement and measurement", Physica D, vol. 120, no. 1-2, pp. 62-81, 1998.

[5] I. Csiszár, J. Körner, Information Theory: Coding Theorems for Discrete Memoryless Systems, Academic Press, New York, 1981.

[6] A. El Gamal, T. Cover, "Multiple User Information Theory", Proc. IEEE, vol. 68, pp. 1466-1483, 1980.

[7] A. S. Holevo, "Bounds for the quantity of information transmitted by a quantum channel", Probl. Inf. Transm., vol. 9, no. 3, pp. $177-183,1973$.

[8] A. S. Holevo, "Problems in the mathematical theory of quantum communication channels", Rep. Math. Phys., vol. 12, no. 2, pp. 273-278, 1977.

[9] A. S. Holevo, "The Capacity of the Quantum Channel with General Signal States", IEEE Trans. Inf. Theory, vol. 44, no. 1, 1998, 269-273.

[10] M. Huang, Y. Zhang, G. Hou, "Classical capacity of a quantum multiple-access channel", Phys. Rev. A, vol. 62, 052106, 2000.

[11] L. B. Levitin, "Quantum Generalization of Conditional Entropy and Information", in: Quantum computing and quantum communications (Palm Springs, CA, 1998), pp. 269-275, LNCS 1509, Springer, Berlin, 1999.

[12] M. Ohya, D. Petz, Quantum Entropy and Its Use, Springer Verlag, Berlin, New York, 1993.

[13] M. Ohya, "Some aspects of quantum information theory and their application to irreversible processes", Rep. Math. Phys., vol. 27, no. 2, pp. 19-47, 1989.

[14] B. Schumacher, "Quantum Coding", Phys. Rev. A, vol. 51, no. 4, pp. 2738-2747, 1995.

[15] B. Schumacher, W. Westmoreland, "Sending classical information via noisy quantum channels", Phys. Rev. A, vol. 56, no. 1, pp. 131-138, 1997.

[16] C. E. Shannon, "Two-way communication channels", in: Proc. Fourth Berkeley Symposium Probability and Statistics (ed. J. Neyman), Berkeley 1961, pp. 611-644. Reprinted in: Claude Elwood Shannon Collected Papers (eds. N. J. A. Sloane, A. D. Wyner), New York 1993, pp. 351-384.

[17] A. Winter, Coding Theorems of Quantum Information Theory doctoral dissertation, Universität Bielefeld 1999, http://archiv.ub.uni-bielefeld.de/disshabi/mathe.htm Also as e-print http://arXiv.org/abs/quant-ph/9907077.

[18] A. Winter "Coding Theorem and Strong Converse for Quantum Channels", IEEE Trans. Inf. Theory, vol. 45, no. 7, pp. 24812485, 1999. 